\title{
Intentional minds: a philosophical analysis of intention tested through fMRI experiments involving people with schizophrenia, people with autism, and healthy individuals
}

\author{
Bruno G. Bara ${ }^{1,2 *}$, Angela Ciaramidaro ${ }^{1}$, Henrik Walter ${ }^{3}$ and Mauro Adenzato ${ }^{1,2}$ \\ Department of Psychology, Center for Cognitive Science, University of Turin, Turin, Italy \\ 2 Neuroscience Institute of Turin, University of Turin, Turin, Italy \\ ${ }^{3}$ Department of Psychiatry and Psychotherapy, Charité Universitätsmedizin Berlin, Berlin, Germany
}

Edited by:

Ivan Toni, Radboud University,

Netherlands

Reviewed by:

Ivan Toni, Radboud University,

Netherlands

Roel M. Willems, University of

California Berkeley, USA

*Correspondence:

Bruno G. Bara, Department of

Psychology, Center for Cognitive

Science, University of Turin, via Po 14

10123 Turin, Italy.

e-mail:bruno.bara@unito.it
In this paper we show how we empirically tested one of the most relevant topics in philosophy of mind through a series of $\mathrm{fMRI}$ experiments: the classification of different types of intention. To this aim, firstly we trace a theoretical distinction among private, prospective, and communicative intentions. Second, we propose a set of predictions concerning the recognition of these three types of intention in healthy individuals, and we report the experimental results corroborating our theoretical model of intention. Third, we derive from our model predictions relevant for the domain of psychopathological functioning. In particular, we treat the cases of both hyper-intentionality (as in paranoid schizophrenia) and hypo-intentionality (as in autistic spectrum disorders). Our conclusion is that the theoretical model of intention we propose contributes to enlarge our knowledge on the neurobiological bases of intention processing, in both healthy people and in people with impairments to the neurocognitive system that underlies intention recognition.

Keywords: theory of mind, communicative intention, prior intention, fMRI, schizophrenia, autism, medial prefrontal cortex, social cognition

\section{PAPER'S GOAL}

The aim of this paper is to analyze how empirical research, specifically neuroimaging studies, can address what have been traditionally thought as a philosophical question, i.e., the classification of different types of intention. The ability to recognize others' intentions is essential for any social interaction. In order to study intentional minds, here we refer to the philosophical approach to intentionality (Searle, 1983) and to a cognitive science approach to communication (Bara, 2010).

Our strategy will be as follows. In Section "Different Types of Intention," we present a taxonomy of intentions, derived essentially by philosophy of mind (here represented by Grice and Searle) and by cognitive pragmatics (Bara). In Section "Predictions for Intentional Minds of Healthy Individuals," we propose a set of experimental predictions on the brain regions involved in processing the different kinds of intention presented in the taxonomy, based on the taxonomy itself and on evolutionary assumptions, as well as on our previously assessed neuroscientific results. In Section "Predictions for Intentional Minds of People with Schizophrenia" we introduce additional hypotheses based on published evidence on schizophrenia research. This allows us to generate further predictions to be tested on people with paranoid schizophrenia, who show hyper-intentionality, i.e., assuming intentionality also where there is none. If people with schizophrenia have a hyper-intentional mind, people with autism may be considered hypo-intentional, as we shall discuss in Section "Predictions for Intentional Minds of Patients with Autism." People with autism, as far as intentional processing is concerned, appear to be located at the opposite extreme than people with schizophrenia, and this allows the generation of a third set of predictions. Here our analysis will be based on a qualitative interpretation of our preliminary data.

\section{DIFFERENT TYPES OF INTENTION}

The first distinction we need is provided by Searle (1983), and is that between prior intention and intention-in-action. Searle regards the intention-in-action (or motor intention) as the direct cause of an agent's movement: the mental and causal component of the bodily movement of an action. To cover the overall conditions of an action one has to analyze also the prior intention, which orients the action as a whole. Prior intention is formed in advance, to represent goal states that may be quite distant from the chain of actions that lead to their fulfillment: prior intention represents the goal of the action before it is undertaken.

A schematic representation of the relation between motor and prior intentions is represented in Figure 1. This figure represents the causal chain from the point of view of the Actor, who is planning an action in order to achieve a goal. The causal chain has to be reversed from the point of view of the Observer, who first recognizes the Actor's bodily movements, then her motor intention, and finally gets to attribute to her a prior intention (see Figure 2). In this paper we shall focus only on the recognition and attribution of prior intentions from a third person's perspective.

\section{PRIOR INTENTIONS IN THE BRAIN}

An exhaustive classification of prior intentions is beyond the scope of this paper (for a wider analysis see Pacherie, 2000, 2006; Becchio et al., 2006). Figure 3 shows the three kind of prior intentions we have tested through fMRI.

Private intentions (PInt) require only the Actor to perform actions adequate to reach her goal (e.g., A intends to drink a glass of water). On the contrary, a social intention requires at least a Partner, who is necessary for the Actor to reach her goal (e.g., A intends 

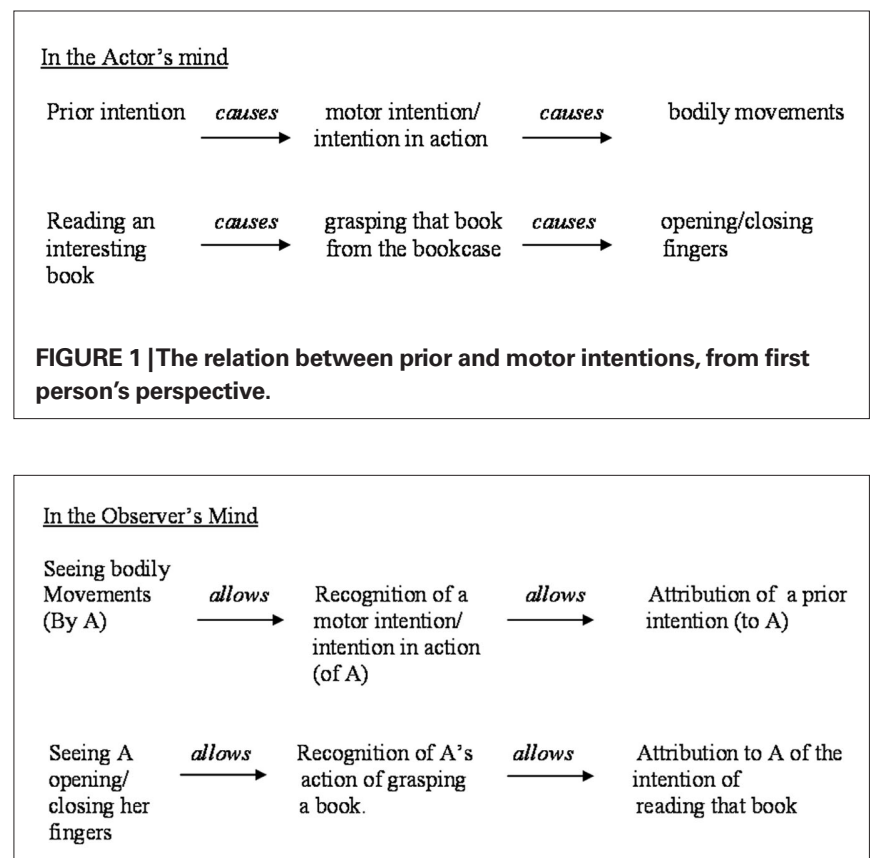

FIGURE 2 |The relation between prior and motor intentions, from third person's perspective.

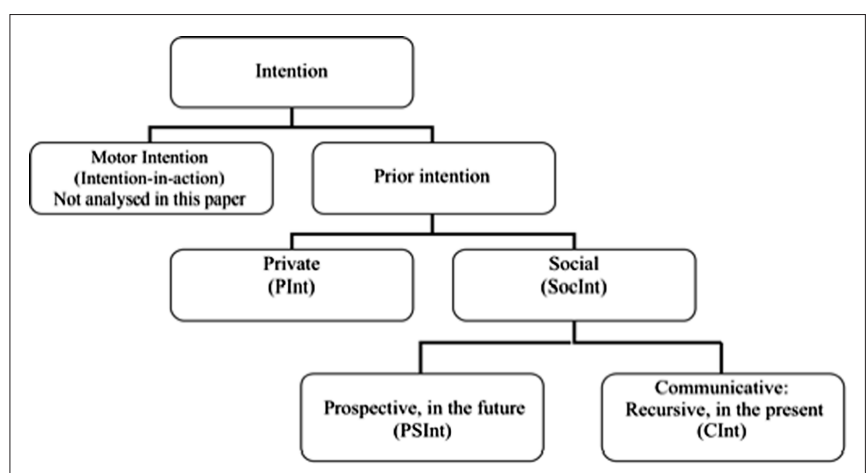

FIGURE 3 | Classification of the different types of prior intention.

to play tennis with B). The notion of shared intention offered by Tomasello (2008) is in principle similar to our social intention, but we go a step further. In our approach, the prototypical case of a social intention shared in the present is Communicative intention (CInt). CInt is the intention to communicate a meaning to someone else, plus the intention that the former intention should be recognized by the addressee (Grice, 1975; Bara, 2010). To be precise, $\mathrm{A}$ intends to communicate that $p$ to $\mathrm{B}$ when $\mathrm{A}$ intends the following two facts to be shared by both A and B:

1. That $p$.

2. That $\mathrm{A}$ intends to communicate that $p$ to $\mathrm{B}$.

Making information achieve the status of sharedness does not mean one has communicated it. For instance, Anne can, in apparent distraction, leave on the table the keys of her Harley-Davidson, thus allowing Barbara to infer that Anne drives such a motorbike. Anne and Barbara now share the fact that Anne has a Harley-Davidson, but Anne can reasonably claim she has never communicated such piece of information to Barbara. The necessary condition for real communication to take place is that such information be intentionally and explicitly proposed to the interlocutor. For this reason, CInt possess the special feature of recursivity (Bara, 2010).

However, there are special intentions whose social goal lies in the future. For instance, Alice may intend to visit her father next Sunday. This kind of social intention involves the representation of a social goal when A and B are not actually interacting but B is part of A's goal. We define this kind of intention Prospective social intention (PSInt). In the case of PSInt, the desired social interaction is not present at the moment, but if the PSInt will become shared in the future, then it will cause the social interaction to occur.

According to Bratman's (1987) account, the main characteristic of future-directed intention (as he prefers to call prior intention) is that the Actor does not only want to accomplish that intention, but she is also committed to do it. If Alice forms the prior intention to visit her father next Sunday, she is committing herself now to visit him on Sunday. Pacherie and Haggard (2010) call all kinds of prior intentions prospective intentions, because they have to be realized in a future moment. For the sake of simplicity, in this paper we reserve the term prospective intention only to social ones (PSInt), even though in principle also private intentions may be future-directed, e.g., Alice may now form the prospective private intention to give up smoking on her next birthday.

In the following sections, we show how we empirically tested the classification presented in Figure 3.

\section{PREDICTIONS FOR INTENTIONAL MINDS OF HEALTHY INDIVIDUALS}

Human beings are constantly involved in various types of social interaction. They cooperate; they compete; they communicate to construct the meaning of their interaction together; they acquire new skills by imitating other people; and they share knowledge, experience, and emotions. In fact, unlike other species, human beings have evolved the ability to regulate social interactions by representing the intentions that motivate other people's behavior. Yet, the complex nature of the interpersonal patterns in which we play an active part on a daily basis is frequently obscured by the apparent ease with which we manage to cope with such complexity.

One of the most currently debated issues is whether the cognitive and neural processes involved in perception, language, memory, and attention, actually suffice to account for the ways in which we conduct our social interaction, or whether we possess mental processes and neural structures that are specifically allocated for this purpose (Adolphs, 2003; Frith, 2007). Our approach follows this latter line of research and assumes that human beings have developed specific brain structures and cognitive mechanisms to cope with social complexity. These mechanisms make it possible to go beyond the surface level of other people's behavior and infer the mental states which generate that behavior. Key abilities involved in these processes are: recognizing other individuals as being goaldirected and intentional agents, and being able to share individual mental states in order to develop a common social space. 
Our predictions on the role played by different brain structures in comprehending intentions are based at first on the data according to which primates present a surprisingly large brain size, especially the prefrontal cortex, in proportion to body weight. Various hypotheses have been put forth to explain the evolution of this phenomenon. The most acclaimed states that the growth of brain volume is an adaptive trait primates developed in response to selective pressures imposed by their complex social systems (Humphrey, 1983). A species' social complexity has been shown to be a function of its social group size (Dunbar, 2009). Groups with greater social interaction require brains with a greater capacity to elaborate social information. Our species has both the highest index of social complexity and the largest prefrontal cortex (Dunbar, 1998; Semendeferi et al., 2001). Our hypothesis proposes that the natural selection has determined the evolution of a mechanism specifically specialized in comprehending mental states which guide people involved in social interaction. In particular, because of the assumed co-evolution of prefrontal cortex size and social relationships, we predict that prefrontal cortex is only recruited in understanding social intentions (i.e., PSInt and CInt). We believe that this specialized mechanism is one of the basic components of the theory of mind (ToM) ability, i.e., the ability both to attribute mental states different from our own to other individuals, and to predict and explain the behavior of others based on these mental states (Premack and Woodruff, 1978). Studies using neuroimaging methods showed the existence of a neural network underlying the ToM ability, including the right and left temporoparietal junctions (TPJ), the precuneus, and the medial prefrontal cortex (MPFC; Fletcher et al., 1995; Brunet et al., 2000; Ruby and Decety, 2003; Saxe and Kanwisher, 2003; Ciaramidaro et al., 2007; Gobbini et al., 2007; Enrici et al., 2011. For a review see Carrington and Bailey, 2009). Converging evidence comes from neurodegenerative and brain lesion studies (e.g., Stuss et al., 2001; Apperly et al., 2004; Adenzato et al., 2010; Poletti et al., 2011).

In performing our $\mathrm{AMRI}$ experiments our main predictions were threefold: (1) the MPFC areas involved in intention recognition are activated only in response to social stimuli requiring comprehension of social interaction, both present and future; (2) the posterior areas underlying intention recognition (TPJ and precuneus) might suffice for representing the mental states of agents, as long as those agents are acting outside social interaction; (3) the existence of a dynamic brain network encompassing the standard ToM regions, i.e., the right and left TPJ, the precuneus and the MPFC, each of which with a specific function depending on what type of prior intention is represented from an observed action.

\section{EXPERIMENTAL EVIDENCE WITH HEALTHY INDIVIDUALS}

In these years research on the neural correlates of social cognition, defined as the set of cognitive processes subserving social behavior in response to conspecifics, are continuously increasing (Amodio and Frith, 2006; Van Overwalle, 2009). Within the realm of social cognition, special attention has been given to the study of the neural mechanism subserving ToM function. This trend of research has permitted the identification of the previously described areas of the brain which together permit the comprehension of the mental states of others, i.e., the TPJ, the precuneus, and the MPFC. Nonetheless, studies of the ToM's neural correlates often have not taken into account that distinct areas of this system can be specialized in the processing of distinct classes of social stimuli. In order to attempt to test our predictions according to which the comprehension of social prior intentions specifically recruits the prefrontal areas of the ToM's neural system, we devised an experimental protocol depicting intentions concerning agents in situations of both social interaction and isolation.

Thus, in a first fMRI experiment (Walter et al., 2004) we presented to healthy participants a series of comic strips (each one of them consisting of a sequence of three pictures) encompassing both "social" and "non-social" stories, i.e., stories that refer, or not, to social interactions. This protocol allowed us to compare the activation of the brain in three different conceptual categories, corresponding with the following comic strip stories: (1) private intentions (PInt) stories, each of which depicts a private action performed by a single character with a private goal, and whose understanding requires the attribution of a private prior intention to the character involved; (2) CInt stories, each of which depicts an action performed by a character with a social goal that is shared with someone else in the present, and whose understanding requires the attribution of a CInt to the character involved; (3) physical causality (Ph-C) stories, a high-baseline condition that does not involve ToM abilities and whose stories depict a non-intentional causal sequence of events concerning objects, whose understanding does not require the attribution of mental states to others. A fourth experimental condition (PInt-2) involving two agents acting independently (not involved in a social interaction) was introduced to control the number of persons involved in the story. The participant were required to choose among a set of three answer pictures the most appropriate and logical continuation of each story (see Figure 4).

In accordance with our a priori experimental hypothesis, the results of this experiment showed that the MPFC (in particular the anterior paracingulate cortex) was significantly activated in the CInt but not in the PInt condition. Thus, our results demonstrated the MPFC is activated only for the representation of intentions involved in social interaction. An alternative explanation for our results, namely the varying number of persons between the CInt and the PInt conditions, was ruled out by our control condition PInt-2: there was no MPFC activation in this condition (see Figure 5).

In order to comprehend to what extent the MPFC is specialized in the understanding of social interaction, we devised a second experiment (Walter et al., 2004). The objective of this experiment was to understand whether the MPFC is active not only in the presence of actual social interaction, such as communicative ones, but also when social interaction is not ongoing at the moment, i.e., when it is only potential. In fact, the results of the first experiment raised the following questions: to what extent can the MPFC activation we found be attributed to the specificity of the communicative interaction? Would the representation of a social goal not involving a CInt have resulted in similar activations? To answer these questions in the second experiment we introduced the PSInt condition (see Figures 3 and 4). In these comic strips a single character who is performing an action which was aimed at intended future social interaction is shown. Thus, the main difference between the CInt and the PSInt condition is that in the latter one the character is not involved in actual social interaction with another character. For example, a character preparing a romantic dinner. 


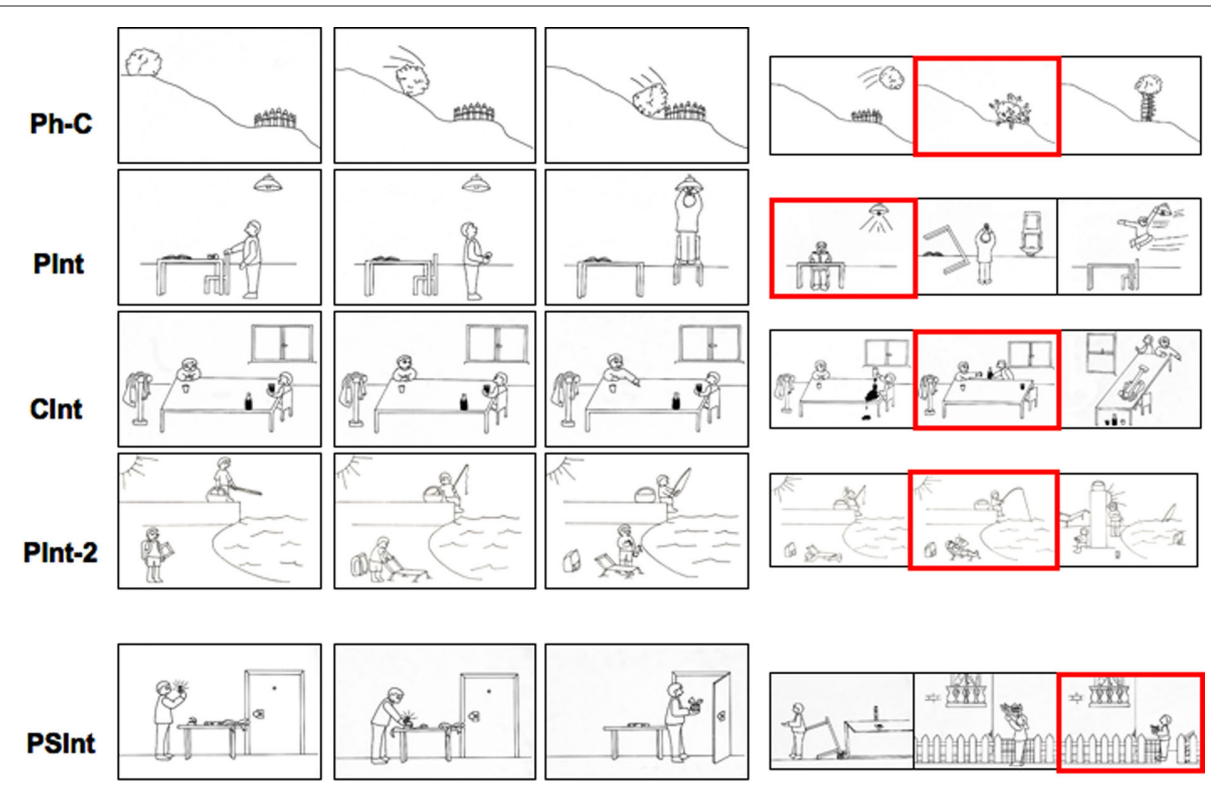

FIGURE 4 | Activation paradigm. One trial for each experimental condition is shown. Red square indicates the correct answer.

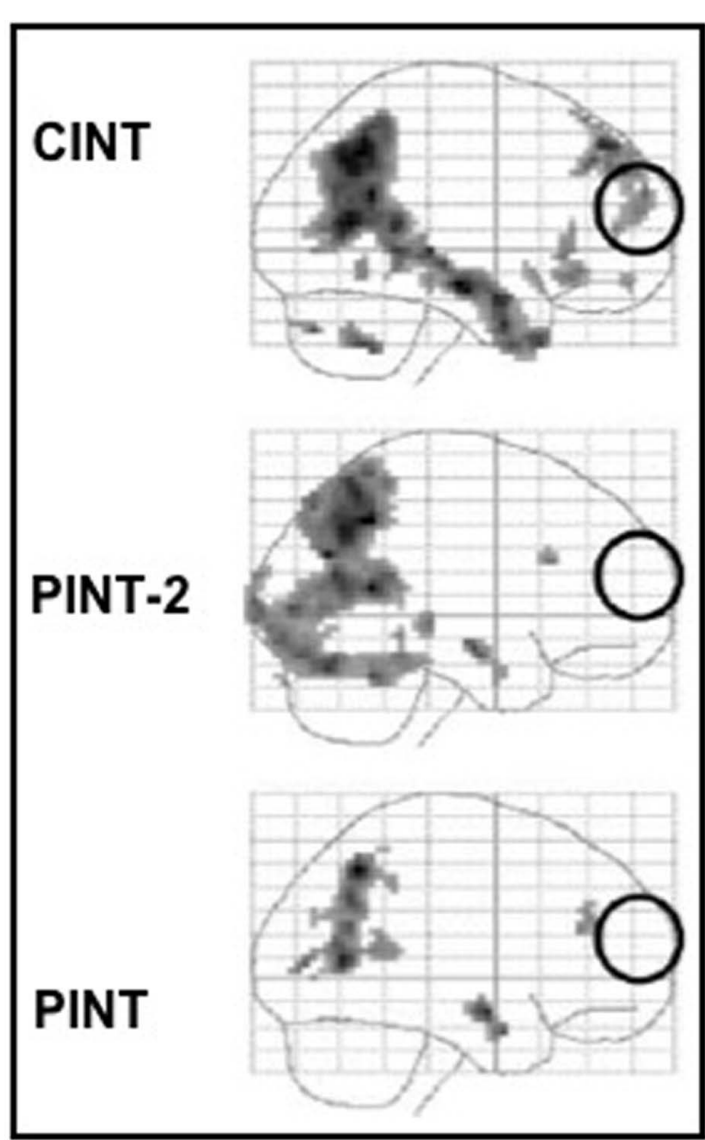

FIGURE 5 | Main effects for the three experimental conditions (PInt, PInt-2, Clnt) vs control condition (Ph-C). Second level, one sample $t$-test, $p<0.001$ uncorrected. Ph-C, Physical causality; PInt, Private intention; PInt-2, Private intentions by two agents; CInt, Communicative intention.
The results of this second experiment replicated the results of the first one with a different and independent sample, i.e., the MPFC was significantly activated in the CInt condition but not in the PInt condition, and in this latter condition posterior brain areas showed to be sufficient to represent the mental states of agents acting outside a social interaction. Furthermore, the results showed for the first time that the MPFC is also engaged in the comprehension of intentions in which a social goal is not yet present but lies in the future, such as in PSInt condition (see Figure 6).

An interesting result we found is a pattern of activations in the MPFC (CInt > PSInt > PInt) that shows the possibility to parameterize the dimension of "socialness" in performing fMRI experiments as recently recommended by Adolphs (2010; see also Figure 7).

Taken together, our experiments with healthy people showed that the MPFC is involved in the comprehension of the intentions involved in social interactions (such as CInt and PSInt) but not in the comprehension of intentions of a person acting outside social interaction (i.e., PInt).

The rationale of the Walter et al.'s (2004) experiments was that the crucial aspect in order to determine whether two different mental states (e.g., CInt and PInt) can be distinguished from a philosophical point of view, is the possibility to find separate neural activations corresponding to each of them, as in the case where the two mental states are processed in spatially distinct locations of the brain. At the same time, however, a large number of high order cognitive functions are neuroanatomically distributed, especially the social cognitive ones (Tamietto et al., 2007; Adolphs, 2010; Botzung et al., 2010): this means that different brain regions are responsible, and work in concert, to process different aspects of the same function. For this reason in a subsequent work (Ciaramidaro et al., 2007) we proposed a model of a dynamic intentional network consisting of the bilateral TPJ, the precuneus, and the MPFC. Our hypothesis was that the intentional network shows different activation patterns in relation to the nature of the prior intentions participants are dealing 


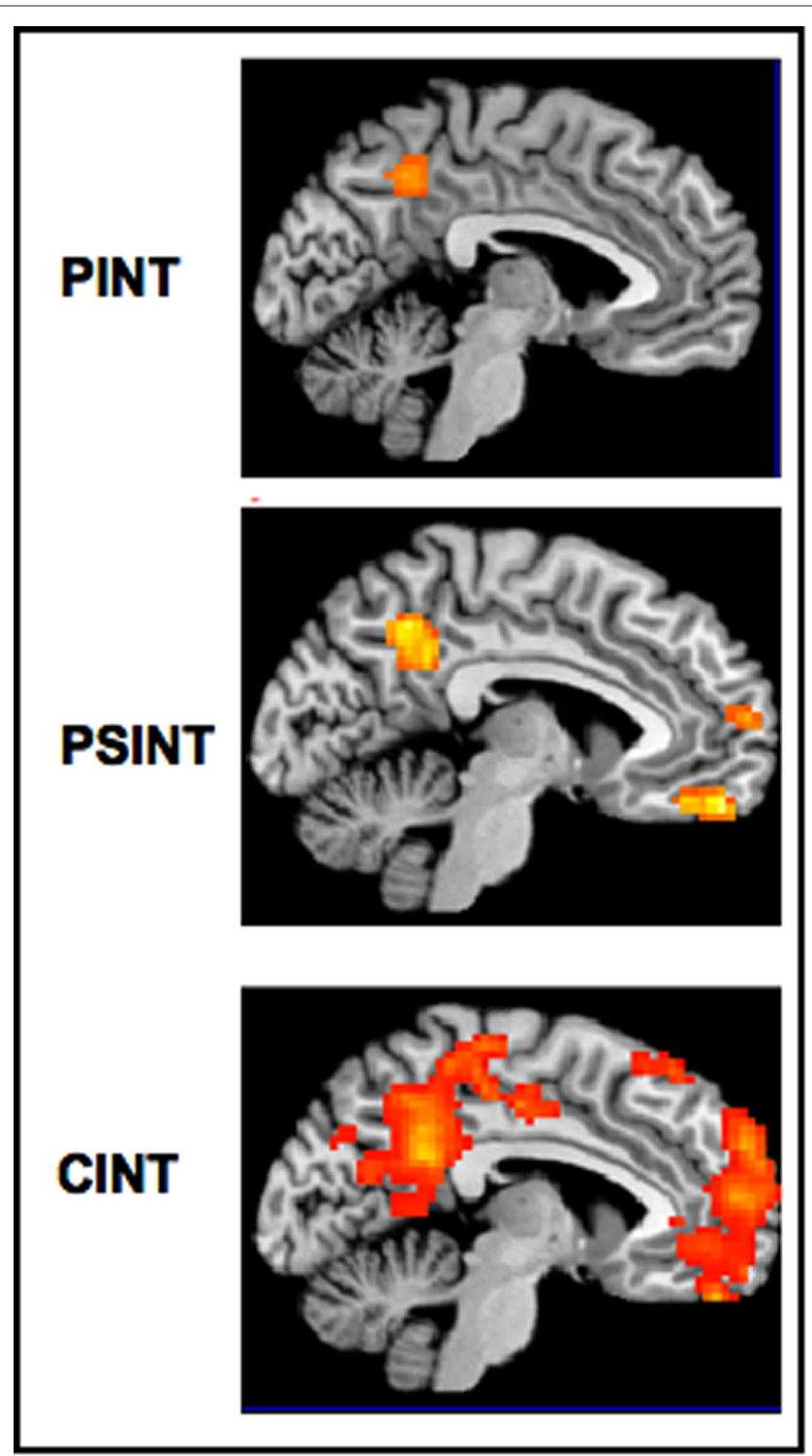

FIGURE 6 | Main effects for the three experimental conditions (PInt, PSInt, Clnt) vs control condition (Ph-C). Second level, one sample $t$-test, $p<0.001$ uncorrected. Ph-C, Physical causality; PInt, Private intention; PSInt, Prospective social intention; CInt, Communicative intention.

with. In particular, we predicted that the network becomes more extensively activated whenever people are trying to comprehend social intentions, i.e., PSInt and CInt conditions.

To test our prediction we extended our earlier results (Walter et al., 2004) by performing a new data analysis that focuses not only on the MPFC, but also on the precuneus and the TPJ bilaterally. This deeper level of analysis was based on signal time courses for the four regions of interest. We found the following main results: (1) only the right TPJ and the precuneus are recruited for the comprehension of private prior intentions (PInt); (2) the recruitment of the right TPJ and the precuneus does not suffice when a person is dealing with a social intention to be shared in the future (PSInt); as hypothesized, it is the MPFC that plays a crucial role in processing this type of intention; (3) the recruitment of the entire neural network underlying ToM is present only when participants were dealing with a shared in the present social intention, i.e., with CInt. This latter result implies a role for left TPJ in understanding CInt, a role we explained considering the well known role of the left hemisphere in communication processing.

In sum, our results show that whereas the right TPJ and the precuneus are necessary for processing all types of prior intentions, the left TPJ and the MPFC are specifically involved in the understanding of social prior intention. More specifically, the left TPJ is activated only when social prior intentions occur in the present (i.e., CInt).

These results demonstrate the progressive recruitment of the intentional network along the theoretical dimensions presented in Section "Different Types of Intention." In the next sections we will discuss further predictions that integrate our taxonomy with clinical evidence, and we test them in two clinical populations, i.e., people with schizophrenia and people with autism.

\section{PREDICTIONS FOR INTENTIONAL MINDS OF PEOPLE WITH SCHIZOPHRENIA}

As human beings we are equipped with the predisposition to attribute mental states to our conspecifics but not to non-living systems; when we see a person communicating with something that is not a living system we immediately register that something peculiar is happening with this individual. Patients with schizophrenia have been shown to exhibit various dysfunctions in the domain of social cognition (Burns, 2004, 2006). In particular, ToM has constantly been found to be impaired in schizophrenia as summarized by two recent meta-analyses comprising 29 ToM studies including 831 patients (Sprong et al., 2007) and 36 ToM studies including 1181 patients (Bora et al., 2009), respectively. ToM dysfunction has been used to explain persecutory delusions (Harrington et al., 2005), a key symptom of schizophrenia characterized by misattribution of intentions to others. It has been argued that patients with delusions of persecution overattribute causes to external events (Bentall et al., 2001) and may be characterized by hyper-intentionality, a specific attitude of paranoid patients which consists in over-attributing significance and intentions to events, persons, and objects. Abu-Akel and Bailey (2000) speak also about "hyper-ToM," an attitude associated with quantitative over-generation of hypotheses or overattribution of mental states. Although paranoid patients seem to experience other people as possessing mental states, they are impaired in using contextual information, an impairment which induces them to make incorrect "online" inferences about others (Brüne, 2005).

Even though there is clear evidence for behavioral deficits in ToM tasks in schizophrenia (for review see Brüne, 2005), the existing studies do not distinguish among different types of mental states or intentions attributed. Also in the few neuroimaging studies investigating ToM in schizophrenia (reviewed in Brunet-Gouet and Decety, 2006) different types of intention were mixed within the same conditions, precluding any specific conclusion about the type of intentional process that might be disturbed. Furthermore, to our knowledge, no neuroimaging study has explicitly investigated the concept of hyper-intentionality in schizophrenia. 


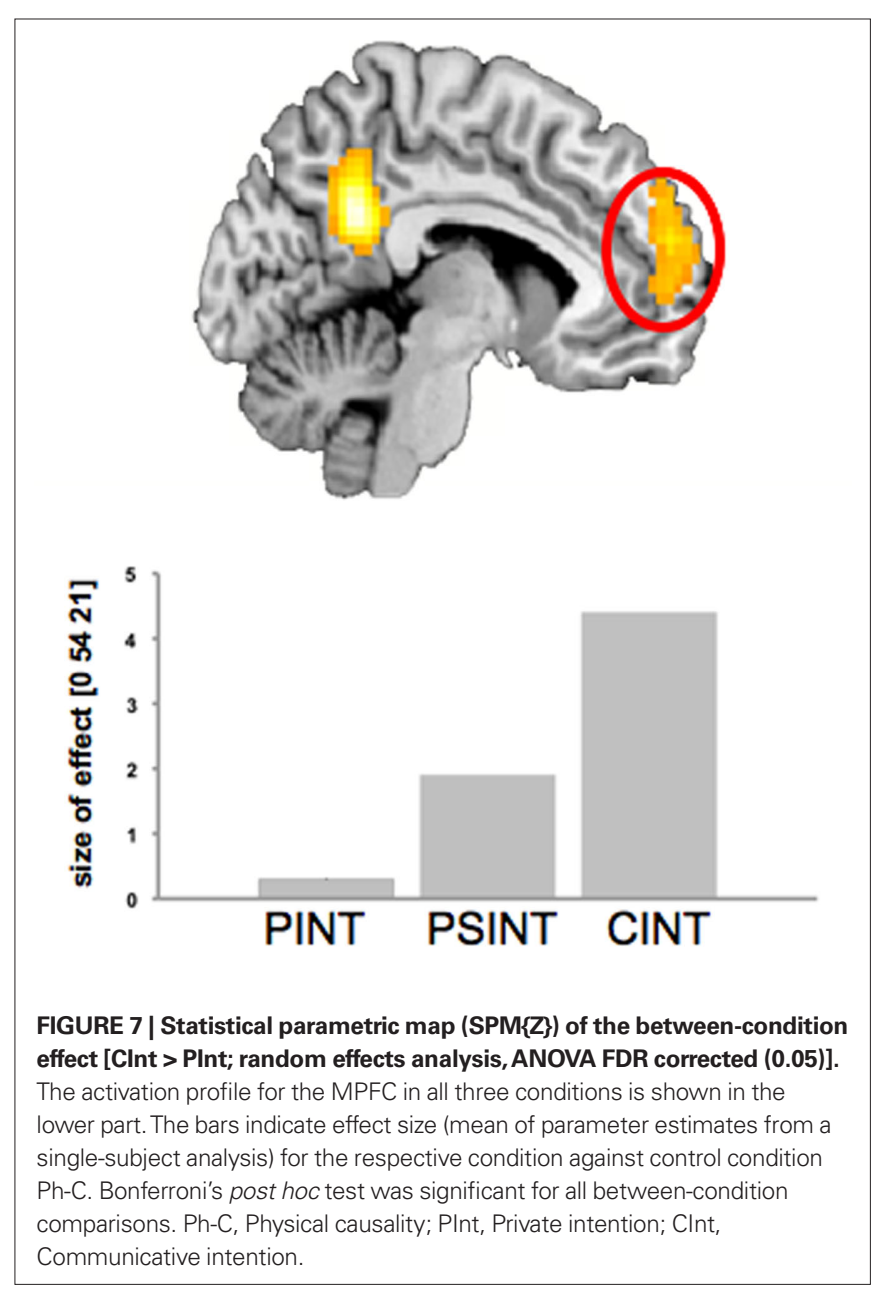

Our model of a dynamic intentional network, described in the previous section, allows us to postulate different activation patterns in relation to the nature of the prior intentions that healthy participants are dealing with. Hence, these results legitimate us to employ this model in order to investigate the maladaptive attribution of intention in paranoid patients and permit us to examine whether their (possible) dysfunctional activation within the intentional network depends on the kind of prior intention involved (private or social). It has been pointed out that ToM deficits in schizophrenia might explain some of the communication problems of these patients (Frith, 2004). Paranoid schizophrenic patients make significantly more mistakes on tasks that involve inferring the beliefs and intentions of the speakers (Tenyi et al., 2002). Langdon et al. (2002), using a picture sequence task, demonstrated pragmatic deficits of expressive language and pragmatic deficits of comprehension, as well as an over-interpretation of linguistic and visual input in patients with schizophrenia (Langdon et al., 2002; see also Russell et al., 2000; Langdon and Coltheart, 2004). These results lead us to hypothesize differential dysfunction in the intentional network, in particular for CInt compared to other types of intentions.

In addition, our experimental model is also indicated to verify in schizophrenic patients "hyper-ToM" attitude to overattribute not only intentions to persons but also to objects: using the control condition of this model ( $\mathrm{Ph}-\mathrm{C})$, we are able to detect whether patients with positive symptoms have a "hyperactive intention detector" which is activated also by physical events. An exaggerated sense of agency seems to characterize patients with delusions of persecution, and this tendency to perceive agency where there is none may be a more general feature of schizophrenia (Frith, 2004). Blakemore et al. (2003) reported that patients with delusions of persecution, when observing simple animations, attributed intentionality to moving shapes in conditions where healthy controls reported no intentional behavior. Therefore, we hypothesized that a similar process would happen in the Ph-C stories.

In our third experiment with schizophrenia patients our main predictions were threefold: (1) we expected to find significant impairments at the behavioral level since performance in attribution of intentions is reduced in schizophrenic patients (Brunet et al., 2003) - the event-related design of the study allowed to analyze correct answers as well as reaction times; (2) we predicted to find a dysfunction in the network underlying ToM in terms of reduced brain activation in the intentional conditions, in particular for CInt, because these patients' attitude of "over-attributing" intentions seems to be related to violations of pragmatic rules (Brüne, 2005); (3) we expected to find neural evidence for the above mentioned hypothesis that paranoid patients may have a hyperactive intention detector when they observe physical events, i.e., in our Ph-C condition they do not deactivate their intention detector.

EXPERIMENTAL EVIDENCE IN PEOPLE WITH PARANOID SCHIZOPHRENIA

Schizophrenia is a heterogeneous disorder with various symptom subgroups showing different degrees of mentalizing impairment. In particular, paranoid patients show ToM deficits because of their incapacity to monitor other people's intentions (Frith, 1992; Frith and Corcoran, 1996; Corcoran et al., 1997). Patients with schizophrenia show considerable deficits in social cognition but the results in the literature are inconsistent. Harrington et al. (2005) mentioned two factors that may explain these inconsistent data: variety of ToM tasks and presence of heterogeneous subgroups of schizophrenic patients included in the studies. For this reason it is recommended to design studies that involve only homogeneous groups of patients and to establish the psychometric properties of the ToM tasks used (Harrington et al., 2005; Sprong et al., 2007). Therefore, we decided to include in our study (Walter et al., 2009) only patients with paranoid schizophrenia. Using the same design with three types of intentions as in our previous studies described in Section "Predictions for Intentional Minds of Healthy Individuals," we compared a group of 12 patients with a matched healthy control group. As already described, the participants' task was to choose the one picture showing the logical ending of a story. Participants indicated their choice by pushing one of three buttons. Eleven comic strips were presented for each of the four conditions described in the Section "Experimental Evidence with Healthy Individuals."

As expected, we found reduced accuracy and increased reaction times for the patient group compared to the control group. However, the reduced performance included all four conditions, i.e., also the control condition ( $\mathrm{Ph}-\mathrm{C})$. These results could have two explanations: (a) they reflect an unspecific impairment in 
the patient group; (b) they reflect a consequence of the patients' "hyper-ToM." "Hyper-ToM" may lead these patients to attribute intentions and goals also to objects, i.e., also during our control condition (Ph-C). In order to better distinguish between these two explanations, in future studies it would be helpful to include an additional control condition not related to causal attribution, to test for impairments depending on more general cognitive deficits.

In our study, the control group showed an increasing activation of the intentional network (private $<$ prospective $<$ CInt) replicating the results from the second experiment of the previous section (Walter et al., 2004). In the patient group there was no MPFC activation in either condition. Comparing groups directly, significant differences in activation patterns were found in the right and left TPJ region and in the MPFC. Our main result, in line with our hypothesis, was that the neural dysfunction of the intentional network was modulated by the type of intention. In particular, for the PInt vs Ph-C conditions, we found no group differences. On the neural level, patients with schizophrenia presented no neural dysfunction for this type of intention. However, the contrast PSInt vs Ph-C revealed significant group differences in the right TJP and the MPFC. Although both intentions (PInt and PSInt) share a common element, namely, one agent acting in isolation, only PSInt requires the representation of a social goal. Also for the CInt comparison there was a group difference in the activation of the left TJP (together with the right TJP and the MPFC). As already described in the previous section, the left TJP was specifically activated for CInt. Taken together, our neuroimaging results present clear evidence that the dysfunctions in the intentional network of paranoid patients are modulated by the different kinds of intention considered: paranoid patients show a reduced activation of the intentional network exclusively during the comprehension of social intentions. No alterations were found during the comprehension of private intentions. We suggest that these patients exhibit this atypical pattern of activation because they are not able to decouple, i.e., to distinguish between the inner mental world and the external world. It has been suggested that the MPFC is the brain region underlying the decoupling mechanism (Gallagher and Frith, 2003). Thus, schizophrenia patients seem unable to distinguish between intentions of others interacting and their own intentions.

Furthermore, we propose that mentalizing impairments in schizophrenia are not only due to a diminished activation in the intentional network, but also to a specific attitude that these patients show, namely their hyper-intentionality. Hyper-ToM is not only intended as an attitude to over-attribute intentions to persons, but also as a tendency to perceive agency where there is none (Frith, 2004). The control condition used in our paradigm (i.e., Ph-C) allowed us to check for this further question. Hyper-intentionality should be accompanied by a lack of deactivation of the intention detector when patients were solving stories involving $\mathrm{Ph}-\mathrm{C}$. The analysis of the beta parameters during all four conditions, showed evidence for this idea, as the lack of differential activation in the MPFC and in the left TPJ was not only due to decreased activation in these regions during the CInt condition, but also to increased beta values in the $\mathrm{Ph}-\mathrm{C}$ condition (see Figure 8).

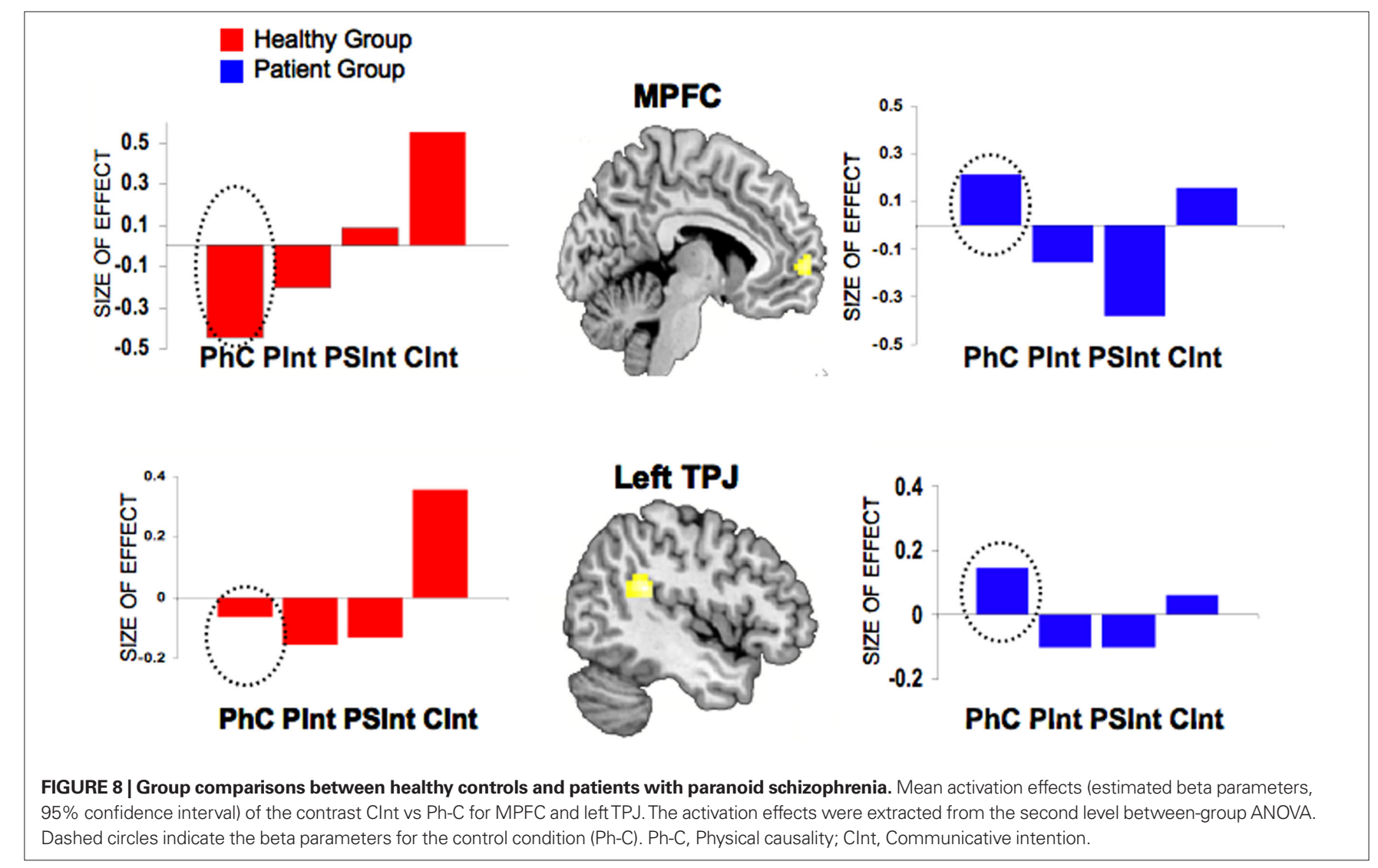


Our results are consistent with the idea that patients with schizophrenia are more or less permanently in an "online" modus of ToM mainly in contexts in which no real intentional agent is involved and in which no ToM is required for solving the task. A similar result was also found by Blakemore et al. (2003). These authors proposed that patients with schizophrenia perceive agency where others see none. This could be the reason why patients make as many errors in the $\mathrm{Ph}-\mathrm{C}$ condition as in the intentional conditions and explains why patients with schizophrenia do not recognize the difference between stories involving intentional agents from stories without agents: also moving objects are processed as possessing intentional agency.

Within our third study we integrated a theory based on philosophy of mind (the taxonomy of intentions) with clinical evidence (the relationship between mentalizing impairment and paranoid symptoms). Such integration allowed us to test our predictions in patients with schizophrenia. In the next section, we develop a similar approach for another clinical group with ToM deficits, i.e., people with autism.

\section{PREDICTIONS FOR INTENTIONAL MINDS OF PATIENTS WITH AUTISM}

Autism is a heterogeneous disorder with multiple causes and courses and this lead autism experts to coin the term "autistic spectrum disorder" (ASD) to underline that this pathology includes different subgroups of patients who differ in terms of the pattern of deficits displayed. ASD is diagnosed on the basis of early emerging social and communicative impairments and rigid and repetitive patterns of behavior and interests. People with ASD show striking limitations in social relatedness and their interest to interact with other people is often absent; they appear distant and display an egocentric attitude also after having learnt basic social rules. They have restricted interests and may exhibit stereotyped behavior and obsessive tendencies. Their ability to communicate is usually compromised and their speech tends to be poor and impersonal (Frith, 2001). Population studies have shown that the prevalence of autism (in its wide range of manifestations) is currently estimated between 0.3 and $0.7 \%$ and that the male to female proportion is approximately three to one (Fombonne, 1999).

Language and communication impairments are so central to ASD that they have become one of the key symptoms necessary to diagnose autism (Tager-Flusberg, 1993). People with ASD do not show a profound deficit in many areas of language (e.g., morphology, lexis, syntax), but they often exhibit severe impairments in communication (e.g., comprehension of ironic expressions). Whereas there is clear behavioral evidence for qualitative impairments in social interaction and communication in ASD, relatively little is known about the neural dysfunction underlying these deficits. Functional imaging techniques provide a new tool to explore this issue.

One hypothesis to explain social deficits in ASD is that they are the result of a specific difficulty in mindreading. People with ASD show deficits in the perception of eye gaze, poor eye contact during communication, and difficulties accessing information to infer the mental state of others. Frith (2001) and Baron-Cohen
(1995), two of the leading experts in autism research, speak about "Mindblindness" in ASD. Abnormalities in brain areas primarily involved in social cognition have been reported by different studies investigating social processing in patients with autism (Brothers, 1990; Baron-Cohen et al., 1999; Castelli et al., 2002; Pelphrey et al., 2002; Klin et al., 2003; Schultz et al., 2003; Gervais et al., 2004; Dalton et al., 2005; Zilbovicius et al., 2006). For example, Baron-Cohen et al. (1999) asked individuals with ASD to judge people's inner states from photographs of their eye region, and to decide which of two words best described their emotional state. Compared to the control group, people with autism demonstrated less extensive activation in frontal regions and no activation in the amygdala. In another study, Castelli et al. (2002) investigated the brain activation of individuals with autism and healthy controls using geometric shapes animations. Two triangles moved about on a screen in three different conditions: moving randomly, moving in a goal-directed fashion (chasing or fighting) and moving interactively with implied intentions (coaxing or tricking). Not only MPFC seems to be a region involved in ASD social dysfunction, the ASD group showed less activation in regions normally involved in social perception, e.g., the posterior temporal sulcus (pSTS). Using fMRI, Gervais et al. (2004) also discovered that individuals with autism failed to activate the pSTS while listening a human voice. The pSTS seems to be clearly implicated in ASD (for review see Zilbovicius et al., 2006) and this region is highly connected with other regions of the "social brain" such as the fusiform face area (FFA), an important area also abnormally activated in ASD during tasks implicating social cognition (Critchley et al., 2000; Schultz et al., 2000; Hubl et al., 2003).

The literature investigating social dysfunction in ASD has also provided an alternative approach to the mindreading hypothesis: the "broken mirror" theory (Williams et al., 2001; Iacoboni and Dapretto, 2006; Ramachandran and Oberman, 2006), which was formulated following the discovery of a class of neurons in monkeys that fire when an action is either executed or observed. A similar mechanism seems to exist also in the human brain and is supported by two main brain regions: the inferior section of the precentral gyrus plus the posterior part of the inferior frontal gyrus (IFG), and the inferior parietal lobule, including the cortex located inside the intraparietal sulcus. These regions form the so called mirror neurons system (MNS; for a recent review see Rizzolatti and Sinigaglia, 2010). This mechanism seems to provide one of the neural bases that connects individuals to each other in form of social interactions (Adenzato and Garbarini, 2006; Brüne and Brune-Cohrs, 2006; Hari and Kujala, 2009). It has been suggested that an impairment in this mechanism may be one of the salient deficits that impair the ability of individuals with ASD to interact with other people: MNS is principally involved in imitation, and the dysfunction of the MNS in ASD might be mainly responsible for a failure in imitation and, because of this, contribute to the later difficulty in understanding others' intentions and emotions.

To sum up, there are two current competitive theories on the origins of the social deficits observed in ASD: the "mindblindness theory" and the "broken mirror" theory, both concerned with the 
atypical patterns of neural activation observed in individuals with ASD when processing social cognition tasks. The brain regions involved by these two hypotheses have distinct but complementary functions for the recognition of others' intentions (De Lange et al., 2008). However, Hamilton (2009) sustains that neither of these two approaches is sufficient for explaining all the difficulties shown by people with ASD; she concludes that a better formulation of the current theories and better empirical data discriminating precisely between goals, intentions, outcomes, and desires are needed. We agree with Hamilton, and we think that our theoretical distinction among varieties of intention could contribute to understand more clearly the social brain dysfunction in ASD. In fact, checking into the MNS experimental paradigms, we found that these studies often implied actions like "grasping, reaching, drinking, cleaning" (e.g., Boria et al., 2009). This kind of stimuli fully corresponds with those probed by our PInt condition. In contrast, most of the stimuli used by the "mindblindness theory" implicate short stories with interacting people or animated shapes (e.g., Castelli et al., 2002), i.e., our CInt condition. Thus, the different paradigms adopted by the two approaches could also be read as diverging in that they use different types of intention (private or social), and this could explain why they linked the social brain dysfunction of ASD to different brain areas. We believe that our model may contribute to the debate on the neural dysfunction involved in social cognition in ASD. In particular, we predict that adopting a paradigm separating social from private intentions, it would be possible to identify different dysfunctional brain regions in patients with autism: "ToM region" like MPFC for social intentions, and IFG (a mirror neurons area) for private intentions. Actually, our group does not yet have final data that may validate this assumption, and further studies on the recognition of different types of intention are needed. However, our model proposes an innovative perspective that may help us to refine our theories of social dysfunction in ASD.

The development of brain mapping methods, their application to normal subjects and their clinical use with patients have had a profound impact on our understanding of normal brain function in healthy individuals and of its alteration in patients with mind/ brain disorders, i.e., patients with autism and schizophrenia. In an exhaustive review, Crespi and Badcock (2008) positioned autistic and psychotic spectrum disorders at the opposite ends of a continuum of cognitive architectures ranging from mechanistic to mentalistic cognition. These authors claimed that people with autism have reduced mentalistic skills (i.e., they are "hypo-mentalistic"), while people with schizophrenia displays opposite features (i.e., they are "hyper-mentalistic"). Adopting a similar approach, we claim that the impairments in understanding others' intentions exhibited by paranoid patients and autistic patients, respectively, can be considered as the two extremes of a continuum.

We believe that our model may enlarge the knowledge on the neurobiological bases of intention processing, and of its relation to social cognition in various forms of psychopathology. By following this line, researchers and clinicians may be able to better understand the nature of neuropsychological and psychopathological deficits in these disorders, and hopefully to formulate more adequate clinical descriptions.

\section{CONCLUSION}

Different domains of investigation may sometimes cross-fertilize each other in order to promote progress in general knowledge. In this paper we presented the case of the interplay between philosophy of mind and cognitive neuroscience.

We regard such cross-fertilization as a fruitful exchange between different epistemological approaches to knowledge, offering it as a paradigmatic case of modern science strategy. We used fMRI experiments with healthy individuals to test the predictions of a theory about prior intentions well assessed both in philosophy of mind and in cognitive science. Then, we used results obtained from people with psychopathological impairments and choose as extremes the cases of autism and paranoid schizophrenia to test the theoretical predictions we derived by philosophy of mind and strengthened through neuroscientific investigation on normal subjects.

Brook (2009) distinguishes between philosophy in cognitive research and philosophy of cognitive research. When philosophy plays a role in cognitive science, it is usually philosophy of mind and language: Brook tout court refers to it as cognitive philosophy. On the generate-and-test picture of science, Brook adds a third activity, interpretation, to the popperian context of hypothesis discovery (big theories) and hypothesis justification (experiments). In this paper, we used philosophy of mind and language for hypothesis discovery (i.e., the taxonomy sketched in Figure 3), then we summarized a series of fMRI experiments dedicated to justify the taxonomy presented. The interpretation of the results allowed us to draw a broad picture encompassing the recognition of intentions in healthy and pathological mind brains.

As a final methodological note, we acknowledge that although neuroimaging techniques provide important information on the involvement of one or more brain areas in the performance of a specific cognitive process, currently available neuroimaging methods do not allow for clear conclusions on whether the same areas are actually required to perform the cognitive task related to the cognitive process examined, i.e., whether they are not only involved but also necessary. Therefore, in accordance with this point of view, our future efforts to test our theory will be based on convergent investigation based on different methodological methods, such as the analysis of how people with real (neuropsychological) and virtual brain "lesions" (the latter induced by Transcranic Magnetic Stimulation) performed in our experimental protocols. These investigations will help us to reach more robust conclusions on the functional role of the brain areas involved in the intentional network and on the taxonomy of different types of intention herein presented.

\section{ACKNOWLEDGMENTS}

We would like to thank Cristina Becchio, Andrew Brook, and Claudia Chiavarino for valuable comments to an early version of the manuscript. This work was supported by University of Turin (Ricerca scientifica finanziata dall'Università 2008 "Correlati cognitivi e neurali della cognizione sociale") and by Regione Piemonte (Project: Institutions, Behavior, and Markets in Local and Global Settings). 


\section{REFERENCES}

Abu-Akel,A., and Bailey, A. L. (2000). The possibility of different forms of theory of mind. Psychol. Med. 30, 735-738.

Adenzato, M., Cavallo, M., and Enrici, I. (2010). Theory of Mind ability in the behavioural variant of frontotemporal dementia: an analysis of the neural, cognitive, and social levels. Neuropsychologia 48, 2-12.

Adenzato, M., and Garbarini, F. (2006). The as if in cognitive science, neuroscience and anthropology: a journey among robots, blacksmiths, and neurons. Theory Psychol. 16, 747-759.

Adolphs, R. (2003). Investigating the cognitive neuroscience of social behavior. Neuropsychologia 42, 119-126.

Adolphs, R. (2010). Conceptual challenges and directions for social neuroscience. Neuron 65, 752-767.

Amodio, D. M., and Frith, C. D. (2006). Meeting of minds: the medial frontal cortex and social cognition. Nat. Rev. Neurosci. 7, 268-277.

Apperly, I. A., Samson, D., Chiavarino, C., and Humphreys, G. W. (2004). Frontal and temporo-parietal lobe contributions to theory of mind: neuropsychological evidence from a falsebelief task with reduced language and executive demands. J. Cogn. Neurosci. $16,1773-1784$

Bara, B. G. (2010). Cognitive Pragmatics. TheMentalProcesses of Communication. Cambridge, MA: MIT Press.

Baron-Cohen, S. (1995). Mindblindness: An Essay on Autism and Theory of Mind. Cambridge, MA: MIT Press.

Baron-Cohen,S., Ring, H.A., Wheelwright, S., Bullmore, E. T., Branner, M. J. Simmons, A., and Williams S. C. (1999). Social intelligence in the normal and autistic brain: an fMRI study. Eur. J. Neurosci. 11, 1891-1898.

Becchio, C., Adenzato, M., and Bara, B. G. (2006). How the brain understands intention. Different neural circuits identify the componential features of motor and prior intentions. Conscious Cogn. 15, 64-74.

Bentall, R. P., Corcoran, R., Howard, R., Blackwood, N., and Kinderman, P. (2001). Persecutory delusions: a review and theoretical integration. Clin. Psychol. Rev. 21, 1143-1192.

Blakemore, S. J., Sarfati, Y., Bazin, N., and Decety, J. (2003). The detection of intentional contingencies in simple animations in patients with delusions of persecution. Psychol. Med. $33,1433-1441$

Bora, E., Murat, Y., and Pantelis, C. (2009). Theory of mind impairment in schizophrenia: meta-analysis. Schizophr. Res. 109, 1-9.

Boria, S., Fabbri-Destro, M., Cattaneo, L., Sparaci, L., Sinigaglia, C., Santelli, E., Cossu, G., and Rizzolatti, G. (2009).
Intention understanding in autism. PLoS ONE 4, e5596. doi: 10.1371/ journal.pone.0005596

Botzung, A., LaBar, K. S., Kragel, P., Miles, A., and Rubin, D. C. (2010) Component neural systems for the creation of emotional memories during free viewing of a complex, real-world event. Front. Hum. Neurosci. 4:34. doi 10.3389/fnhum.2010.00034

Bratman, M. E. (1987). Intention, Plans, and Practical Reason. Cambridge, MA Cambridge University Press.

Brook, A. (2009). Introduction: philosophy in and philosophy of cognitive science. Top. Cogn. Sci. 1, 216-230.

Brothers, L. (1990). The social brain: a project for integrating primate behaviour and neurophysiology in a new domain. Concepts Neurosci. 1, 27-51.

Brüne, M. (2005). Emotion recognition, "theory of mind," and social behavior in schizophrenia. Psychiatry Res. 133, 135-147.

Brüne, M., and Brune-Cohrs, U. (2006) Theory of mind - evolution, ontogeny, brain mechanisms and psychopathology. Neurosci. Biobehav. Rev. 30, 437-455.

Brunet, E., Sarfati, Y., Hardy-Baylé, M.-C., and Decety, J. (2000). A PET investigation of the attribution of intentions with a nonverbal task. Neuroimage 11, 157-166.

Brunet, E., Sarfati, Y., Hardy-Baylé, M. C., and Decety, J. (2003). A PET study of the attribution of intentions to others in schizophrenia. Neuropsychologia 41 1574-1582.

Brunet-Gouet, E., and Decety, J. (2006) Social brain dysfunctions in schizophrenia: a review of neuroimaging studies. Psychiatry Res. 148, 75-92.

Burns, J.K. (2004). An evolutionary theory of schizophrenia: cortical connectivity, metarepresentation, and the social brain. Behav. Brain Sci. 27, 831-885.

Burns, J. K. (2006). Psychosis: a costly by-product of social brain evolution in Homo sapiens. Prog. Neuropsychopharmacol.Biol.Psychiatry 30, 797-814.

Carrington, S. J., and Bailey, A. J. (2009). Are there theory of mind regions in the brain? A review of the neuroimaging literature. Hum. Brain Mapp. 30 2313-2335.

Castelli, F., Frith, C., Happé, F., and Frith, U. (2002). Autism, Asperger syndrome and brain mechanisms for the attribution of mental states to animated shapes. Brain 125 1839-1849.

Ciaramidaro, A., Adenzato, M., Enrici, I., Erk, S., Pia, L., Bara, B. G., and Walter, H. (2007). The intentional network: how the brain reads varieties of intentions. Neuropsychologia 45 , 3105-3113.
Corcoran, R., Cahill, C., and Frith, C. D. (1997). The appreciation of visual jokes in people with schizophrenia. A study of "mentalizing" ability. Schizophr. Res. 24, 319-327.

Crespi, B., and Badcock, C. (2008). Psychosis and autism as diametrical disorders of the social brain. Behav. Brain Sci. 31, 241-261.

Critchley, H., Daly, E., Bullmore, E. Williams, S., Van Amelsvoort, T. Robertson, D., Rowe, A., Phillips, M., McAlonan, G., Howlin, P., and Murphy, D. (2000). The functional neuroanatomy of social behavior: changes in cerebral blood flow when people with autistic disorder process facial expressions. Brain 123 , 2203-2212.

Dalton, K. M., Nacewicz, B. M., Johnstone, T., Schaefer, H. S., Gernsbacher, M. A. Goldsmith, H. H., Alexander, A. L. and Davidson, R. J. (2005). Gaze fixation and the neural circuitry of face processing in autism. Nat. Neurosi. 8, 519-526.

De Lange, F. P., Spronk, M., Willems, R M., Toni, I., and Bekkering, H. (2008). Complementary systems for understanding action intentions. Curr. Biol. 18, 454-457.

Dunbar, R. I. M. (1998). The social brain hypothesis. Evol. Anthropol. 6 178-190.

Dunbar, R. I. M. (2009). The social brain hypothesis and its implications for social evolution. Ann. Hum. Biol. 36 , 562-572.

Enrici, I., Adenzato, M., Cappa, S. Bara, B. G., and Tettamanti, M (2011). Intention processing in communication: a common brain network for language and gestures. J. Cogn. Neurosci. doi: 10.1162/ jocn.2010.21594

Fletcher, P., Happé, F., Frith, U., Baker, S. C., Dolan, R. J., Frackowiak, R. S. J., and Frith, C.D. (1995). Other minds in the brain: a functional imaging study of "theory of mind" in story comprehension. Cognition 57, 109-128.

Fombonne, E. (1999). The epidemiology of autism: a review. Psychol. Med. 29 , 769-786.

Frith, C. D. (1992). The Cognitive Neuropsychology of Schizophrenia. Hove: Lawrence Erlbaum Associates.

Frith, C. D. (2004). Schizophrenia and theory of mind. Psychol. Med. 34 385-389.

Frith, C. D. (2007). The social brain? Philos. Trans. R. Soc. Lond., B, Biol. Sci. 362, 671-678.

Frith, C. D., and Corcoran, R. (1996) Exploring "theory of mind" in people with schizophrenia. Psychol. Med. 26, 521-530.

Frith, U. (2001). Mind blindness and the brain in autism. Neuron 32, 969-979.
Gallagher, H. L., and Frith, C. D. (2003). Functional imaging of "theory of mind." Trends Cogn. Sci. 7, 77-83.

Gervais, H., Belin, B., Boddaert, N., Leboyer, M., Coex, A., Sfaello, I., Barthélémy, C., Brunelle, F., Samson, Y., and Zilbovicius, M. (2004). Abnormal cortical voice processing in autism. Nat. Neurosci. 7, 801-802.

Gobbini, M. I., Koralek, A. C., Bryan, R. E., Montgomery, K. J., and Haxby, J.V. (2007). Two takes on the social brain: a comparison of theory of mind tasks. J. Cogn. Neurosci. 19, 1803-1814.

Grice, H. P. (1975). "Logic and conversation," in Syntax and Semantics. Speech Acts, eds P. Cole and J. L. Morgan (New York: Academic Press), 41-58.

Hamilton, A. (2009). Research review: goals, intentions and mental states: challenges for theories of autism. J. Child. Psychol. Psychiatry 50, 881-892.

Hari, R., and Kujala, M. V. (2009). Brain basis of human social interaction: from concepts to brain imaging. Physiol. Rev. 89, 453-479.

Harrington, L., Langdon, R., Siegert, R. J., and McClure, J. (2005). Theory of mind in schizophrenia: a critical review. Cogn. Neuropsychiatry 10, 249-286.

Hubl, D., Bolte, S., Feineis-Matthews, S., Lanfermann, H., Federspiel, A., and Strik, W. (2003). Functional imbalance of visual pathways indicates alternative face processing strategies in autism. Neurology 61, 1232-1237.

Humphrey, N. (1983). Consciousness Regained: Chapters in the Development of Mind. Oxford: Oxford University Press.

Iacoboni, M., and Dapretto, M. (2006). The mirror neuron system and the consequences of its dysfunction. Nat. Rev. Neurosci. 7, 942-951.

Klin, A., Jones, W., Schultz, R., and Volkmar, F. (2003). The enactive mind, or from actions to cognition: lessons from autism. Philos. Trans. R. Soc. Lond., B, Biol. Sci. 358, 345-360.

Langdon, R., and Coltheart, M. (2004). Recognition of metaphor and irony in young adults: the impact of schizotypal personality traits. Psychiatry Res. 125, 9-20.

Langdon, R., Coltheart, M., Ward, P. B. and Catts, S. V. (2002). Disturbed communication in schizophrenia: the role of poor pragmatics and poor mindreading. Psychol. Med. 32, 1273-1284.

Pacherie, E. (2000). The content of intentions. Mind Lang. 15, 400-432.

Pacherie, E. (2006). "Towards a dynamic theory of intentions," in Does Consciousness Cause Behavior? An Investigation of the Nature of Volition, eds S. Pockett, W. P. Banks, and S. 
Gallagher (Cambridge, MA: MIT Press), 145-167.

Pacherie, E., and Haggard, P. (2010). "What are intentions?" in Benjamin Libet and Agency, eds L. Nadel and W. Sinnott-Armstrong (Oxford: Oxford University Press), 70-84.

Pelphrey, K. A., Sasson, N. J., Reznick, J. S., Paul, G., Goldman, B. D., and Piven, J. (2002). Visual scanning of faces in autism. J. Autism Dev. Disord. 32, 249-261.

Poletti, M., Enrici, I., Bonuccelli, U., and Adenzato, M. (2011). Theory of mind in Parkinson's disease. Behav. Brain Res. doi: 10.1016/j.bbr.2011.01.010. [Epub ahead of print].

Premack, D., and Woodruff, G. (1978). Does the chimpanzee have a theory of mind. Behav. Brain Sci. 1 515-526.

Ramachandran, V. S., and Oberman, L. M. (2006). Broken mirrors: a theory of autism. Sci. Am. 295, 62-69.

Rizzolatti, G., and Sinigaglia, C. (2010). The functional role of the parietofrontal mirror circuit: interpretations and misinterpretations. Nat. Rev. Neurosci. 11, 264-274.

Ruby, P., and Decety, J. (2003). What you belief versus what you think they believe: a neuroimaging study of conceptual perspective-taking. Eur. J. Neurosci. 17, 2475-2480.

Russell, T. A., Rubia, K., Bullmore, E. T., Soni, W., Suckling, J., Brammer, M. J., Simmons, A., Williams, S., and Sharma, T. (2000). Exploring the social brain in schizophrenia: left prefrontal underactivation during mental state attribution. Am. J. Psychiatry 157, 2040-2042.

Saxe, R., and Kanwisher, N. (2003). People thinking about thinking people. the role of the temporo-parietal junction in "theory of mind." Neuroimage 19, 1835-1842.

Schultz, R., Gauthier, I., Klin, A., Fulbright, R., Anderson, A., Volkmar, F., Skudlarski, P., Lacadie, C., Cohen, D., and Gore, J. (2000). Abnormal ventral temporal cortical activity during face discrimination among individuals with autism and Asperger syndrome. Arch. Gen. Psychiatry 57, 331-340.

Schultz, R., Grelotti, D., Klin, A., Kleinman, J., Van der Gaag, C., Marois, R., and Skudlarski, P. (2003). The role of the fusiform face area in social cognition: implications for the pathobiology of autism. Philos. Trans. R. Soc. Lond., B, Biol. Sci. 358, 415-427.

Searle, J. R. (1983). Intentionality. Cambridge: Cambridge University Press.

Semendeferi, K., Armstrong, E., Schleicher, A., Zilles, K., and Van Hoesen, G. W. (2001).Prefrontal cortex in humans and apes: a comparative study of area 10 . Am. J. Phys. Anthropol. 114, 224-241.

Sprong, M., Schothorst, P., Vos, E., Hox, J., and Van Engeland, H. (2007). Theory of mind in schizophrenia: meta-analysis. Br. J. Psychiatry 191, 5-13.

Stuss, D.T., Gallup, G. G.Jr., and Alexander, M. P. (2001). The frontal lobes are nec- essary for "theory of mind." Brain 124, 279-286.

Tager-Flusberg, H. (1993). "What language reveals about the understanding of other minds in children with autism," in Understanding Other Minds, eds S. Baron-Cohen, H. TagerFlusberg, and D. Cohen (New York: Oxford Medical), 138-157.

Tamietto, M., Adenzato, M., Geminiani, G., and de Gelder, B. (2007). Fast recognition of social emotions takes the whole brain: interhemispheric cooperation in the absence of cerebral asymmetry. Neuropsychologia 45, 836-843.

Tenyi, T., Herold, R., Szili, I. M., and Trixler, M. (2002). Schizophrenics show a failure in the decoding of violations of conversational implicatures. Psychopathology 35, 25-27.

Tomasello, M. (2008). Origins of Human Communication. Cambridge, MA: MIT Press.

Van Overwalle, F. (2009). Social cognition and the brain: a meta-analysis. Hum. Brain Mapp. 30, 829-858.

Walter, H., Adenzato, M., Ciaramidaro, A., Enrici, I., Pia, L., and Bara, B. G. (2004). Understanding intentions in social interaction: the role of the anterior paracingulate cortex. J. Cogn. Neurosci. 16, 1854-1863.

Walter, H., Ciaramidaro, A., Adenzato, M., Vasic, N., Ardito, R. B., Erk, S., and Bara, B. G. (2009). Dysfunction of the social brain in schizophrenia is modulated by intention type: an fMRI study. Soc. Cogn. Affect. Neurosci. 4, 166-176.

Williams, J. H., Whiten, A., Suddendorf, T., and Perrett, D. I. (2001). Imitation, mirror neurons and autism. Neurosci. Biobehav. Rev. 25, 287-295.

Zilbovicius, M., Meresse, I., Chabane, N., Brunelle, F., Samson, Y., and Boddaert, N. (2006). Autism, the superior temporal sulcus and social perception. Trends Neurosci. 7, 359-366.

Conflict of Interest Statement: The authors declare that the research was conducted in the absence of any commercial or financial relationships that could be construed as a potential conflict of interest.

Received: 20 July 2010; accepted: 10 January 2011; published online: 02 February 2011.

Citation: Bara BG, Ciaramidaro A, Walter $H$ and Adenzato M (2011) Intentional minds: a philosophical analysis of intention tested through fMRI experiments involving people with schizophrenia, people with autism, and healthy individuals. Front. Hum. Neurosci. 5:7. doi: 10.3389/ fnhum.2011.00007

Copyright $\odot 2011$ Bara, Ciaramidaro, Walter and Adenzato. This is an open-access article subject to an exclusive license agreement between the authors and Frontiers Media SA, which permits unrestricted use, distribution, and reproduction in any medium, provided the original authors and source are credited. 